\title{
Carbothermal and boron carbide reduction of oxides of some transition metals
}

\author{
Yuriy L. Krutskii ${ }^{1}$, Tatiana M. Krutskaya ${ }^{2}$, Tatiana S. Gudyma ${ }^{1-}$, Konstantin B.Gerasimov ${ }^{3}$, \\ Roman R. Khabirov ${ }^{1}$, and Anna V. Mass ${ }^{1}$ \\ ${ }^{1}$ Novosibirsk State Technical University, 20, K. Marx Prospect, Novosibirsk, Russia, 630071 \\ ${ }^{2}$ Novosibirsk State University of Architecture and Civil Engineering, 113, Leningradskaya St., \\ Novosibirsk, Russia, 630008 \\ ${ }^{3}$ Institute of Solid State Chemistry and Mechanochemistry, SB RAS, 18, Kutateladze St., Novosibirsk, \\ Russia, 30090
}

\begin{abstract}
The study presents a possible mechanism to produce carbides and diborides of transition metals, such as titanium, vanadium, chromium and zirconium. The carbothermal synthesis of transition metal carbides has defined the direct dependence between the thermodynamic stability of oxides and the temperature range of the reduction onset (the stronger the oxide, the higher the value of the temperature is). It reaches 2000-2100, $1500-1600,1300-1400$ and $2100-2200^{\circ} \mathrm{C}$ for such carbides as $\mathrm{TiC}, \mathrm{VC}_{0,88}$, $\mathrm{Cr}_{3} \mathrm{C}_{2}$ and $\mathrm{ZrC}$ respectively. The same dependence has not been found for the diborides of these metals. Optimum synthesis temperatures for all these compounds lie in the range of $1600-1700{ }^{\circ} \mathrm{C}$. This viable method to produce transition metal carbides consists in the transfer of vaporous higher and lower oxides. Diborides preparation involves the transfer of oxides and boron vapors onto the surface of the carbon material with the subsequent chemical interaction. In the case of carbide-boron reduction of zirconium oxide in excess of boron carbide, the reaction product will be a composite material $\left(\mathrm{B}_{4} \mathrm{C}-\mathrm{ZrB}_{2}\right)$. The ceramics based on this composite possesses high performance properties.
\end{abstract}

\section{Introduction}

Carbides and diborides of certain transition metals (titanium, vanadium, chromium, and zirconium) find wide application in state-of-the art technologies both in the form of powder and compact products owing to their unique properties, such as refractoriness, high hardness, chemical inertness, etc. Different methods are proposed for their preparation. The most promising techniques applied in production of carbides and diborides are considered to be carbothermal and boron carbide reduction with carbon respectively. The objective of the study is to explain the processes occurring during the synthesis of transition metals carbides by the carbothermal method and diborides by the carbide-boron method.

- Corresponding author: gudymatan@mail.ru 


\section{Experimental}

To do experiments on the synthesis of carbides and diborides of titanium, vanadium, chromium and zirconium, nanofibrous carbon (NFC) was used as a carbon material characterized by a high specific surface area $\left(\sim 150 \mathrm{~m}^{2} / \mathrm{g}\right)$ and a low content of impurities (at the level of 1 wt.\%). The synthesis of titanium, vanadium, chromium and zirconium diborides was carried out in an argon protective atmosphere, which prevented undesirable nitriding of the boron-containing reagent - boron carbide [1].

\section{Results and discussion}

The optimum synthesis temperatures are given in Table 1. The synthesis of titanium, vanadium, chromium, and zirconium carbides has revealed that the values of the optimum production temperatures depend directly on the thermodynamic stability of the oxides used as reagents: $\mathrm{ZrO}_{2}>\mathrm{TiO}_{2}>\mathrm{V}_{2} \mathrm{O}_{3}>\mathrm{Cr}_{2} \mathrm{O}_{3}$ (Fig. 1).

Table 1. Optimum temperatures for the synthesis of carbides and diborides of titanium, vanadium, chromium and zirconium.

\begin{tabular}{|c|c|c|c|}
\hline \multicolumn{4}{|c|}{ Optimum temperatures for the synthesis of carbides, ${ }^{\circ} \mathrm{C}$} \\
\hline $\mathrm{TiC}$ & $\mathrm{VC}_{0,88}$ & $\mathrm{Cr}_{3} \mathrm{C}_{2}$ & $\mathrm{ZrC}$ \\
\hline $2000-2100$ & $1500-1600$ & $1300-1400$ & $2100-2200$ \\
\hline \multicolumn{4}{|c|}{ Optimum temperatures for the synthesis of diborides, ${ }^{\circ} \mathrm{C}$} \\
\hline $\mathrm{TiB}_{2}$ & $\mathrm{VB}_{2}$ & $\mathrm{CrB}_{2}$ & $\mathrm{ZrB}_{2}$ \\
\hline $1600-1700$ & $1600-1700$ & $1600-1700$ & $1600-1700$ \\
\hline
\end{tabular}

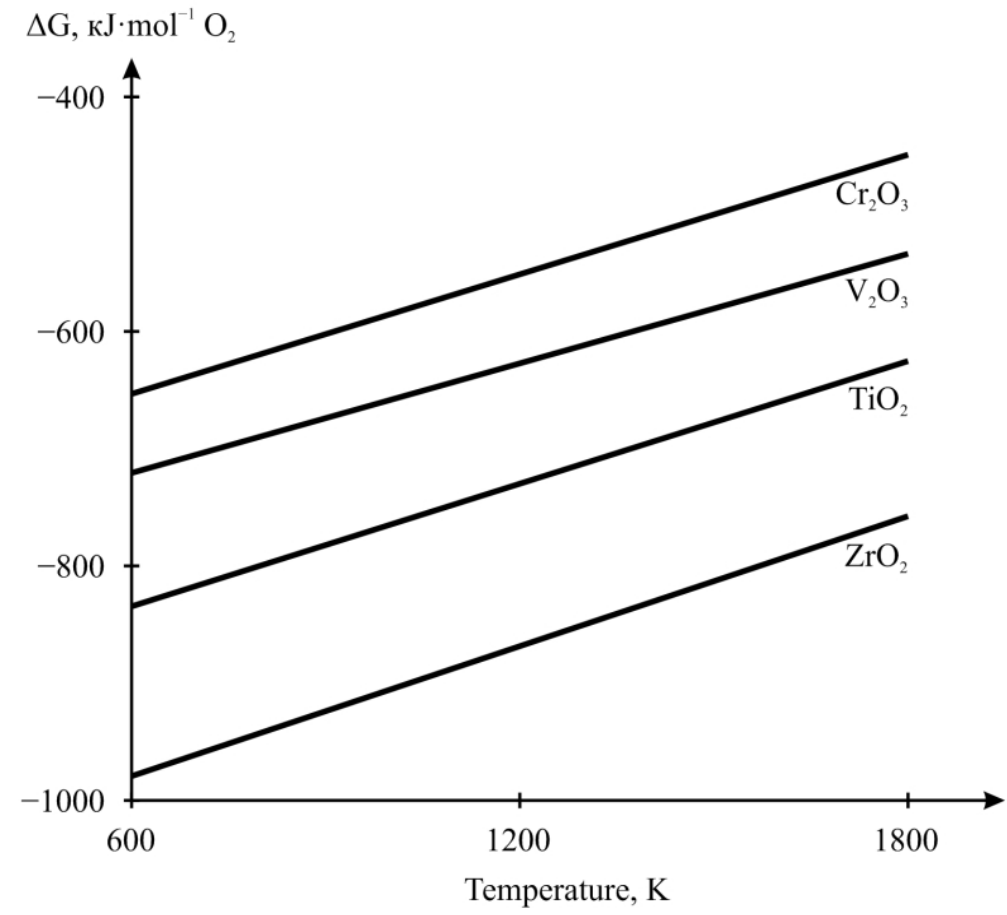

Fig. 1. Temperature dependence of free energy for the following oxides production: $\mathrm{TiO}_{2}, \mathrm{~V}_{2} \mathrm{O}_{3}$, $\mathrm{Cr}_{2} \mathrm{O}_{3}, \mathrm{ZrO}_{2}$ [2]. 
It should be taken into account that the optimum (experimental) temperatures for the synthesis of all the diborides are practically comparable $\left(1600-1700{ }^{\circ} \mathrm{C}\right)$ without regard to the thermodynamic stability of the oxides used as reagents. Apparently, participation of boron carbide in the reaction influences "leveling" of the optimum temperatures to produce the above-mentioned compounds.

Based on the obtained experimental and literature data, it could be possible to propose a mechanism of the refractory compounds formation. The authors consider that the refractory carbides reduction from thermodynamically strong metal oxides with $\mathrm{CO}$ is impossible (the role of carbon is reduced to carbon monoxide (CO) regeneration). The process of carbide production through the interaction of oxides with solid carbon is more probable, as reported in the monograph [3]. They come to the following conclusion based on the experimental data. Although reactions could proceed at the points of direct contact between solid reagents (metal oxides and carbon), the resulting products sharply reduce the rate of the process and limit its development. In this regard, it is hard to explain the significant rates of solid-phase reactions observed in practice owing to the mechanism of direct interaction (contact) between solid reagents. The conclusion obviously suggests itself: gases and substances vapors participate in solid-phase interactions. The transfer of oxide vapors onto the carbon surface of the reducing agent was also observed for the compounds with relatively low vapor pressure. In general, the mechanism of metal oxides interaction with carbon is comprised of the following main stages.

1. Thermal decomposition (sublimation, dissociation) of a solid oxide under formation of a vapor phase being adsorbed on the surface of a solid carbon reducing agent.

2. Chemical interaction of the adsorbed layer with carbon and desorption of the gaseous reaction products $\left(\mathrm{CO}, \mathrm{CO}_{2}\right)$.

It has been experimentally established that the kinetic characteristics of the interaction processes are precisely determined by the physicochemical properties of metal oxides. Therefore, there is no reason to exaggerate the role of the regeneration reaction of carbon monoxide versus $\mathrm{C}+\mathrm{CO}_{2}=2 \mathrm{CO}$ reaction taking place under reduction of metal oxides distinguished by sufficiently high thermodynamic stability. This point of view is confirmed by the following specific data.

It should be noted that information on the possible mechanism of boride formation under the carbide-boron synthesis of borides is not available in the literature. When analyzing the processes, one should proceed from the assumption that carbon vapor pressure is significantly lower compared with vapor pressure of metal oxides and boron within the range of synthesis temperatures. Consequently, carbon vapor pressure reaches: $9 \cdot 10^{-9} ; \sim 2 \cdot 10^{-7} ; \sim 3 \cdot 10^{-6}, \sim 4 \cdot 10^{-5} ; \sim 2.6 \cdot 10^{-3}$ and $\sim 8.5 \cdot 10^{-2} \mathrm{~Pa}$ at temperatures, $\mathrm{K}: 1700$, $1800,1900,2000,2200$ and $2400,2.6 \cdot 10^{-3} \sim 8.5 \cdot 10^{-2}$ respectively [4]. The vapor pressure, observed above used oxides, is as follows [5]. At $300 \mathrm{~K}$ (practically corresponding to the optimum temperature for the synthesis of titanium carbide), vapor pressure of titanium oxide $\left(\mathrm{TiO}_{2}\right)$ reaches $\sim 1 \mathrm{~Pa}$; at $2000 \mathrm{~K}$ (nearly corresponding to the optimum temperature for the synthesis of titanium diboride), it is equal to $\sim 0.01 \mathrm{~Pa}$. The vapor contains $\mathrm{Ti}^{+}$and $\mathrm{TiO}^{+}$ions, $\mathrm{TiO}$ and $\mathrm{TiO}_{2}$ molecules, and $\mathrm{Ti}$ atoms above this oxide. At $1900 \mathrm{~K}$ (practically corresponding to the optimum synthesis temperatures of vanadium carbide and diboride), vapor pressure of vanadium oxide $\mathrm{V}_{2} \mathrm{O}_{3}$ is $\sim 0.1 \mathrm{~Pa}$. The vapor contains $\mathrm{VO}$ and $\mathrm{VO}_{2}$ molecules above this oxide. Chromium oxide $\left(\mathrm{Cr}_{2} \mathrm{O}_{3}\right)$ vapors reach the pressure of $0.001 \mathrm{~Pa}$ at $1600 \mathrm{~K}$ (approximately corresponding to the optimum synthesis temperature of higher chromium carbide); but it equals $\sim 0.1 \mathrm{~Pa}$ at $2000 \mathrm{~K}$ (approximately corresponding to the optimal temperature for the synthesis of chromium diboride). The vapor contains $\mathrm{Cr}$ atoms and $\mathrm{CrO}, \mathrm{CrO}_{2}$ molecules above this oxide. At $2400 \mathrm{~K}$ (approximately equal to the optimum temperature for the zirconium carbide synthesis), the pressure of vaporous zirconium oxide $\left(\mathrm{ZrO}_{2}\right)$ is $0.01 \mathrm{~Pa}$; at $2000 \mathrm{~K}$ (approximately corresponding to the optimal 
temperature for the synthesis of zirconium diboride), it reaches $\sim 0.0001 \mathrm{~Pa}$. The vapor contains predominantly $\mathrm{ZrO}_{2}$ molecules above this oxide.

Boron vapor pressure is known [5] to be $\sim 0.1 \mathrm{~Pa}$ above the boron carbide-carbon system at $2000 \mathrm{~K}$ (approximately corresponding to the optimal synthesis temperatures of the considered transition metals diborides); and the pressure of other gaseous components prove to be lower by two orders of magnitude $\left(\mathrm{BC}_{2}\right)$ and three orders of magnitude $\left(\mathrm{B}_{2} \mathrm{C}\right)$.

Thus, the pressure of oxides and boron vapors significantly exceeds (by several orders of magnitude) the pressure of vaporous carbon at the optimum synthesis temperatures for all refractory compounds. Moreover, based on the case study of carbothermal chromium oxide reduction [7], it was found out that vapor pressure tends to increase above this oxide under conditions of reduction. The same phenomena are likely to occur during other carbothermal and carbide-boron processes.

Therefore, it could be asserted with a high degree of probability that these processes are carried out by means of transferring vapors of higher and lower oxides onto the surface of solid carbon (synthesis of carbides), and via transferring vapors of higher and lower oxides along with vaporous boron onto the surface of solid carbon (synthesis of diborides). These are typical adsorption processes. Relatively short synthesis periods of the considered refractory compounds appear to be an indirect proof of this asumption. Then, diffusion processes will follow on completion of chemical reactions (although they are viable at direct contact of solid reagents). The developed surface of the nanofibrous carbon (NFC) is certain to play a positive role in these relatively fast processes. It obviously reduces the time of diffusion processes, which are completed by full conversion of reagents into target compounds.

In the case of carbide-boron reduction of zirconium oxide with in excess of boron carbide, the reaction product will be a composite material composed of $\mathrm{B}_{4} \mathrm{C}-\mathrm{ZrB}_{2}$. The ceramics based on this composite reveals high performance properties [8].

\section{Conclusions}

The studies resulted in determination of the most possible mechanism aimed at production of transition metal carbides (titanium, vanadium, chromium and zirconium). The method consists in the transfer of oxide vapors, and vaporous diborides - oxide and boron onto the surface of a solid carbon material with the subsequent chemical interaction. The developed surface of the nanofibrous carbon (NFC) is certain to play a positive role.

The study was accomplished in accord with the state order made by the Ministry of Education and Science (FSUN-2020-0008 code).

\section{References}

1. Yu.L. Krutskii, A.G. Bannov, E.V. Antonova, V.V. Sokolov, A.Yu. Pichugin, E.A. Maksimovskii, T.M. Krutskaya, O.V. Netskina, I.A. Bataev. Ceram. Int. 40, 3212 (2017)

2. G.V. Samsonov, Physicochemical properties of oxides (Metallurgiya, Moscow, 1978)

3. V.P. Elyutin, Yu.A. Pavlov, V.P. Polyakov, S.B. Sheboldaev, Interaction of metal oxides with carbon (Metallurgiya, Moscow, 976)

4. G.V. Samsonov, Properties of elements. Part 1 Metallurgiya, (Moscow, 976)

5. E.K. Kazenas, Yu.V. Tsvetkov, Thermodynamics of oxides evaporation (LKI, Moscow, 2008) 
6. A.S. Bolgar, A.G. Turchanin, V.V. Fesenko, Thermodynamic properties of carbides (Naukova Dumka, Kiev, 973)

7. L.-M. Berger, S. Stolle, W. Gruner, K. Wetzig, Int. J. Refract. Met. Hard Mater. 19, 109 (2001)

8. V.A. Shestakov, T.S. Gudyma, Yu.L. Krutskii, N.F. Uvarov. Mater. Today: Proc. 31, 3 (2020) 